\title{
ESTUDIOS DE CASO DE LA INTELIGENCIA EMOCIONAL EN LOS ESTUDIANTES DE GRADO DE EDUCACIÓN
}

\section{CASE STUDIES OF EMOTIONAL INTELLIGENCE IN EDUCATION DEGREE STUDENTS}

Beatriz Peña Acuña. Universidad de Huelva. España.

beatriz.pa@dfilo.uhu.es

María Luisa García Hernández. Universidad de Murcia. España.

luisagarcia@um.es

\section{RESUMEN}

Se trata de una investigación sobre inteligencia emocional llevada a cabo en dos casos de estudio con alumnas universitarias españolas de los Grados de Educación mediante un proyecto con carácter innovador en el que se les introdujo a contenidos de inteligencia emocional, se trabajaba la inteligencia emocional en el aula de forma trasversal identificando las emociones, se les animó a apuntar un diario de notas sobre el clima emocional del aula, a identificar en obras audiovisuales las emociones vividas en el lenguaje corporal junto a los tipos de inteligencia hallados en los personajes, así como la relación de la inteligencia emocional con la inteligencia social con respecto a la expresión y la empatía. Queríamos hacer esta investigación para comprobar la eficacia de las actividades docentes y el interés de las alumnas sobre esta cuestión con el fin de vertebrar con posterioridad un proyecto de innovación sobre inteligencia emocional que fuera financiado. La herramienta de investigación usada ha consistido en un test para que autoevaluaran la inteligencia emocional antes y después de aplicársele la metodología docente para que se conocieran, y en una entrevista semiestructurada que recogía datos cuantitativos y datos cualitativos y de la que extraemos datos.

PALABRAS CLAVE: inteligencia emocional; educación superior; alumnus; clima de aula; emociones; estudio de casos; identificación; expression; gestión emocional.

\section{ABSTRACT}

This is an investigation on emotional intelligence carried out in two cases of study with Spanish university students of the Degrees of Education through a pilot project of innovation in which they were introduced to emotional intelligence content, emotional intelligence was worked on Classroom transverse identifying emotions, they were encouraged to write down a diary of notes on the emotional climate of the classroom, to identify in audiovisual works the emotions experienced in body language along with the types of intelligence found in the characters, as well as the relationship of emotional intelligence with social intelligence. We wanted to do this research to verify the effectiveness of the activities and the interest of the students on this issue in order to structure an innovation project on emotional intelligence that 
was financed. The research tool used was a test to self-assess the emotional intelligence before and after applying the teaching methodology so that they knew each other, and in a semi-structured interview that collected quantitative data and qualitative data and from which we extracted data.

KEYWORDS: emotional Intelligence; student's higher education; environment classroom; emotions; case studies; identification; expression; emotions' management.

Peña Acuña, B. y García Hernández, M. (2018). Estudios de caso de la inteligencia emocional en los estudiantes de grado de educación. Revista de Ciencias de la Comunicación e Información, 23(2), 37-53.

doi: http://doi.org/10.35742/rcci.2018.23(2).37-53

\section{INTRODUCCIÓN}

Tras varios estudios de Peña $(2012,2014,2015,2018)$ sobre inteligencia emocional, la académica estimó conveniente aplicar un proyecto con carácter innovador sobre esta cuestión en alumnos de Educación. Para ilustrar la inteligencia emocional, comenzamos con un acercamiento al concepto, y más aún, lo vinculamos este término con la formación de los futuros docentes. Fue a mediados del siglo XX cuando comenzaron a surgir distintas investigaciones que ayudaron a la conceptualización del término. "En España a pesar de que el estudio de la inteligencia emocional es un aspecto reciente, las investigaciones científicas están experimentando un creciente desarrollo académico" (Sánchez, 2014, p. 193).

Evidentemente, podemos encontrar múltiples y diversas definiciones sobre la inteligencia y, concretamente, sobre la inteligencia emocional. En los orígenes de la conceptualización del término encontramos el trabajo realizado por Salovey y Mayer (1990) quienes afirmaban que "la inteligencia emocional es un subconjunto de la inteligencia social que implica la habilidad de reconocer los sentimientos y las emociones tanto propios como ajenos, discriminar entre ellos y usar esa información para orientar el pensamiento y las acciones" (p.185). Pocos años más tarde, en 1997, ampliaron el concepto, y afirmaron que la inteligencia emocional es:

la habilidad para percibir, valorar y expresar emociones con exactitud, la habilidad para acceder y/o generar sentimientos que faciliten el pensamiento; la habilidad para comprender emociones y el conocimiento emocional y la habilidad para regular las emociones promoviendo un crecimiento emocional e intelectual (p. 4).

Reuven Bar-On (1997) estableció en sus primeros estudios que la inteligencia emocional "es un conjunto de capacidades, competencias y habilidades que desde su condición no cognitiva influyen en la habilidad de un individuo para tener éxito ante las demandas o presiones generadas por el ambiente en el que se desarrolla" (p.36-37).El impulso al concepto de inteligencia emocional y el reconocimiento fuera de la comunidad científica es atribuido a Goleman (1995). Sin duda, la inteligencia emocional pese a ser estudiada desde hace décadas actualmente ha cobrado un inusitado auge. "Cada vez se publican más estudios científicos que confirman la 
estrecha relación entre las emociones, el pensamiento y la toma de decisiones de las personas" (Pedrera y Revuelta, 2016, p. 71). Cabe destacar que, este tipo de inteligencia favorece "el aprendizaje de habilidades sociales y emocionales, está permitiendo a los niños afrontar situaciones, aumentar el autocontrol emocional, autorregularse, generalización de los aprendizajes al ámbito personal y social, mejora de las calificaciones académicas, etc." (Peña, Díez y García, 2012, p. 27). La inteligencia emocional no consiste únicamente en poseer determinadas aptitudes, sino en mucho más: "este concepto apela al desarrollo de la capacidad emocional de las personas, enriquecimiento personal, emocional y social que a su vez repercute en la consecución del bienestar" (Sánchez, 2016, pp. 45-46). Actualmente nos encontramos en un momento de profundo cambio en el modo de interpretar las relaciones interpersonales, "ya sea desde el entorno académico, organizacional o educativo. Se está haciendo cada vez más patente la apuesta por el desarrollo de competencias emocionales tanto para la capacitación de individuos como de equipos profesionales" (Sánchez, 2014, p. 191). Por otra parte, con el desarrollo de la inteligencia emocional se contribuye a uno de los principios fundamentales de la educación, según la Ley Orgánica Española 2/2006, de 3 mayo, como es el de proporcionar una educación de calidad a todos los ciudadanos, conjugando la calidad con la equidad educativa, y también el desarrollo íntegro de la personalidad del individuo (Peña, Díez y García, 2012, p. 29). Fernández de Bobadilla, Peña y Parra (2014) recogen que "la inteligencia emocional ha adquirido un lugar prominente entre las competencias profesionales (...)" (p. 534). Competencias que se deben adquirir a lo largo del proceso de formación de los futuros maestros y que se les solicitarán cuando sean docentes en el aula. Sin duda, "es interesante analizar las emociones y su repercusión en el proceso de enseñanza- aprendizaje (...) ya que nos permite conocer y analizar el estado anímico de los estudiantes" (López de la Serna y Castaño-Garrido, 2018, p. 304). Así pues, si nuestra intención es que los futuros maestros pongan en práctica esta competencia estos autores señalan que, "para acceder a la inteligencia emocional pensamos que es conveniente primero dar relevancia en la formación del alumnado universitario al uso de la técnica de la inteligencia emocional para tener conciencia de las propias emociones, identificarlas y autorregularlas" (Fernández de Bobadilla, Peña y Parra (2014, p. 536). Es evidente que, "con el desarrollo de un modelo teórico, se hace necesario evaluar las dimensiones emocionales que presenta dicho modelo a través de un instrumento de medida" (Pedrera y Revuelta, 2016, p. 75) tal y como analizaremos en el siguiente apartado.

\section{OBJETIVOS}

Presentar un proyecto docente con carácter innovador mediante estudio de casos a grupos de estudiantes de Educación de introducción a la inteligencia emocional en cuanto a identificación de las cinco emociones, expresión de estas y su gestión. Presentar la investigación mixta acerca de la inteligencia emocional de estos estudiantes y de la eficacia de esta intervención.

\section{METODOLOGÍA}

Es una investigación de tipo mixto, tanto cuantitativo a través de preguntas cerradas y de tipo cualitativo a través de preguntas abiertas mediante una entrevista 
semiestructurada. Además, está apoyada por la observación participante que llevo a cabo la profesora que puso en marcha el proyecto sobre inteligencia emocional. La finalidad de esta investigación es conocer la aplicación de la inteligencia emocional en el ámbito profesional educativo en dos estudios de caso tras una puesta en marcha de una intervención con carácter innovador sobre inteligencia emocional en el aula de Educación de Infantil y Primaria. Concretamente nos planteamos la necesidad de que los alumnos se concienciaran de la importancia de la inteligencia emocional tanto en la vida personal, como en la profesional tanto para saber identificar emociones como saber expresarlas a través de un aprendizaje desde la experiencia. Asimismo, esperábamos introducirlos en la gestión emocional aprovechando las ocasiones que se dieran de forma natural en clase, de modo trasversal.

Como hemos avanzado en estos dos estudios de casos (de grupos de alumnos de Educación) se han utilizado datos mixtos (tanto datos cualitativos como cuantitativos) extraídos de una entrevista semiestructurada. Los datos mixtos se cruzan y se extraen conclusiones menores acerca de los dos casos. Otro de los objetivos era valorar la identificación y la gestión emocional de los futuros maestros. Así pues, se les instruyó a los alumnos de modo teórico a través de clases magistrales, documentación subida al campus y la realización de un test personal acerca de la inteligencia emocional en el que se podían autoevaluar respecto a esta capacidad, de este modo, a través de este test disponían de ejemplos concretos de acción que determina una menor o mayor capacidad de identificación y gestión emocional (Peña, Díez y García, 2012, p. 23).

En cuanto a la muestra compuesta por 58 alumnos, indicar que la investigación se ha centrado en la titulación de Grado en Educación Primaria y Grado en Educación Infantil en la modalidad presencial y semipresencial.

Tabla 1. Descripción de la muestra: Titulación.

\begin{tabular}{lc}
\hline & $N^{\circ}$ participantes \\
\hline Grado en Educación Infantil & 15 \\
\hline Grado en Educación Primaria & 14 \\
\hline TOTAL & 29 \\
\hline
\end{tabular}

Fuente: elaboración propia.

Tabla 2. Descripción de la muestra: Modalidad.

\begin{tabular}{lc}
\hline & $N^{\circ}$ participantes \\
\hline Modalidad Semipresencial & 18 \\
\hline Modalidad Presencial & 11 \\
\hline TOTAL & 29 \\
\hline
\end{tabular}

Fuente: elaboración propia.

Si tenemos en cuenta la información que aparece en la tabla $n^{\circ} 1$ observamos que hemos alcanzando un total de 29 estudiantes, de los cuales 15 pertenecían al Grado de Educación Infantil y 14 al Grado de Educación Primaria. En cuanto a la modalidad 
de estudio podemos destacar que 18 han sido estudiantes de la modalidad semipresencial, mientras que 11 pertenecen a la modalidad presencial.

Por otra parte, resaltar que nos preocupó recoger una muestra significativa cualitativa con la que profundizar intentando contar con las experiencias y percepciones de los estudiantes. Si bien entendemos que la recogida de la muestra cuantitativa es insuficiente, pero combinada con la mixta, sin embargo, aporta en este caso cierta luz a los datos cualitativos y está sintonizada, como veremos, en esta investigación. La técnica de recogida de información fue una entrevista semiestructurada, donde había preguntas cerradas (que respondían a una parte cuantitativa de la investigación) y una pregunta abierta (en la que se podía recoger información cualitativa). Este tipo de herramienta mixta como hemos dicho anteriormente nos permite cruzar datos de diversa índole y alcance. Para concluir el apartado metodológico hemos de señalar que se utilizó el paquete estadístico IBM SPSS Statistics 21 para el tratamiento de los datos cuantitativos. Para la realización de este análisis se organizó la información de acuerdo con las categorías consultadas en el cuestionario, estableciendo así las distintas variables.

\section{RESULTADOS}

Vamos a exponer algunos de los resultados más destacados obtenidos en la entrevista semiestructurada sobre Inteligencia Emocional. En cuanto a los resultados cuantitativos, destacar que comenzaremos con el análisis de los datos sociodemográficos (género, edad, titulación y modalidad), continuaremos con el análisis e interpretación de los datos relacionados con los sentimientos hacia la inteligencia emocional (la elaborar un diario de notas sobre el clima emocional de la clase; identificación de las emociones del grupo; elaboración de prácticas vinculadas con las emociones; si se consideran personas de confianza a las que se les puede pedir consejo; cuántos amigos tienen y si en alguna ocasión han realizado un test de inteligencia emocional) y, concluiremos el análisis con comentarios y sugerencias realizadas por los estudiantes sobre la actividad realizada y su experiencia acerca de la inteligencia emocional.

En el primer apartado del análisis profundizaremos en el género, tal y como se observa en la gráfica $n^{\circ} 1$ que se muestra a continuación.

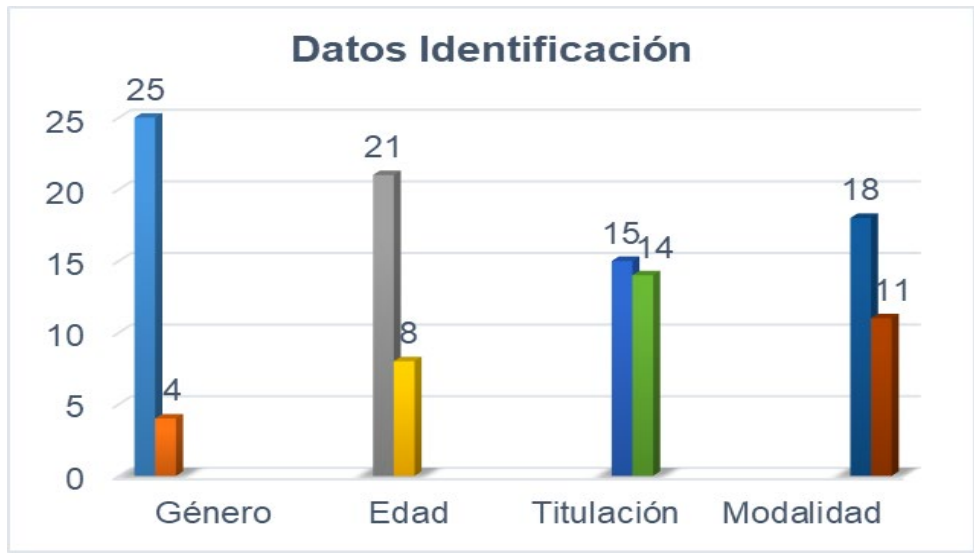

Figura1. Datos de identificación de la muestra participante. 
De acuerdo con la figura $\mathrm{n}^{\circ} 1$ el género de los participantes en la investigación es mayoritariamente femenino ya que han colaborado 25 mujeres $(86,20 \%$ de un total de 29), mientras que sólo 4 eran varones (un 13,80\% del total). Este dato puede estar justificado porque, como veremos más adelante, las mujeres son mayoritariamente las que se matriculan en titulaciones relacionadas con Educación.

Seguidamente, analizaremos los datos relacionados con la edad de los participantes. Tomando como referencia la información obtenida en esta investigación los dos rangos de edad oscilaban entre una cohorte entre 18-23 años y una segunda cohorte entre 23-50 años. Así pues, el número mayoritario de alumnos matriculados en estas titulaciones oscilaba en un 72,4\% entre los entre 23-50 años, ya que 21 de los 29 participantes seleccionaron esta opción. Por otra parte, sólo 8 sujetos participantes (un $27,59 \%$ del total) han marcado que se encuentran en la franja de edad comprendida entre 18-23 años mientras cursan estos Grados.

Seguidamente, profundizaremos en la titulación cursada por los sujetos en el momento de la recogida de información. En cuanto a la titulación en la que están matriculados los estudiantes que han participado en esta investigación, podemos confirmar que 15 de los 29 estudiantes (un 51,72\% del total) estaban estudiando el Grado en Educación Infantil mientras que 14 de 29 (48,27\% del total) cursaban Educación Primaria. Como podemos comprobar no existen diferencias significativas en cuanto a la participación de la muestra entre ambas titulaciones. Por otra parte, y tal y como podemos observar en la gráfica, se hace referencia a la modalidad de estudios de Grado en la que estaban matriculados los sujetos. En este sentido, podemos confirmar que un elevado porcentaje de la muestra productora de datos $(62,07 \%)$ de estaba matriculada en la modalidad semipresencial ya que fueron 18 de 29 los sujetos alumnos que respondieron el cuestionario y que estaban matriculados en esta opción; mientras que el resto de alumnos estaban matriculados en la modalidad presencial $(37,93 \%)$. Hasta el momento hemos descrito e interpretado los datos sociodemográficos e identificativos de la muestra participante en esta investigación. En este sentido y teniendo en cuenta la información aportada podemos confirmar que la muestra mayoritaria era: mujeres, con edades comprendidas entre los 23 y los 50 años, que estudian el Grado de Educación Infantil y, concretamente, en la modalidad semipresencial. Seguidamente, analizaremos los datos relacionados con los sentimientos hacia la inteligencia emocional. Así pues, comenzaremos con la elaboración de un diario de notas y su influencia en el clima emocional de la clase. Esta información queda recogida en la gráfica $\mathrm{n}^{\circ} 2$.

\section{Diario sobre emoción}

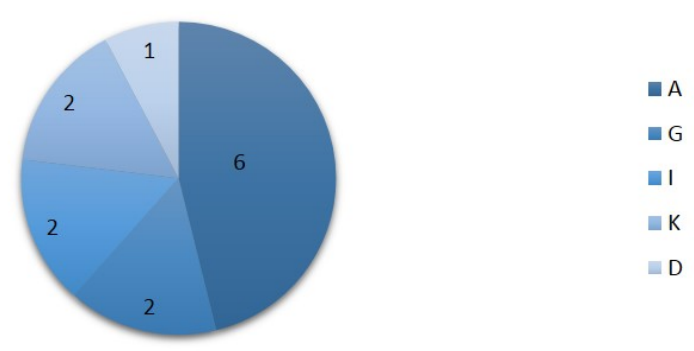

Figura 2. Datos sobre la elaboración de un diario de notas sobre el clima emocional. 
Más concretamente, a la pregunta ¿elaborar un diario de notas sobre el clima emocional de la clase en qué te ha ayudado? Los sujetos podían escoger entre diversas opciones de respuesta como:

A.- En observar más que antes a las personas y percibir más detalles de lenguaje corporal y emociones de los demás.

B.- En aprender a elaborar un diario de notas.

C.- En darle más importancia a mis emociones.

D.- En identificar mis emociones.

E.- En reconocer que tengo esas emociones.

F.- En gestionarlas (por ejemplo, contar un chiste cuando estoy agobiada en un examen)

G.- Me he ayudado en dos respuestas anteriores.

$\mathrm{H}$.- Me he ayudado en tres respuestas anteriores.

I.- Me he ayudado en cuatro respuestas anteriores.

J.- Me he ayudado en todo lo anterior (en las 5).

K.- Ninguna de las respuestas anteriores es mi caso.

Así pues, podemos afirmar que 6 de los 29 participantes $(20,68 \%$ del total) seleccionaron la opción A; seguidamente 2 de los 29 participantes $(6,89 \%)$ señalaron la opción G; del mismo modo, otros 2 participantes escogieron la opción I; y 2 miembros participantes se identificaban con la alternativa K. Por otra parte, 1 sujeto $(3,44 \%$ del total de la muestra) seleccionó la alternativa $D$ y otro escogió la opción J. Por último, señalar que ninguno de los sujetos seleccionó las opciones $B$, C, E, F ni H. Para estos participantes la elaboración de un diario no les ha ayudado a desarrollar estas habilidades.

Seguidamente, analizaremos los resultados obtenidos en la categoría sobre la identificación de las emociones en clase en la gráfica $n^{\circ} 3$ que mostramos a continuación.

\section{Identificación emociones clase}

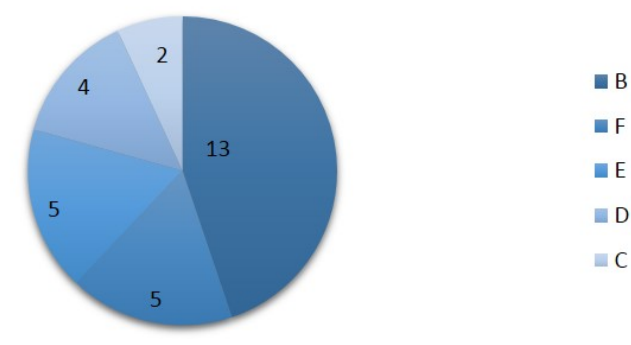

Figura 3. Datos sobre la identificación de emociones en clase.

Así pues, a la pregunta "el hecho de que la profesora durante la clase o tutorías identificara las emociones del grupo cuando se producían", los sujetos podían escoger entre diversas opciones de respuesta entre las que se encuentran:

A.- Me ha ayudado a entender que cada uno tiene emociones que responden a una situación. 
B.- Me ha ayudado a entender que cada uno tiene emociones, pero también nos contagiamos de emociones de forma conjunta (por ejemplo, durante los exámenes toda la clase se pone muy nerviosa y tiene miedo)

C.- Me ha ayudado a identificar qué es el miedo, la ira, la sorpresa, la tristeza y la alegría.

D.- Me ha ayudado en dos respuestas (en una no).

E.- Me ha ayudado en todo lo anterior.

F.- Ninguna de las respuestas anteriores es mi caso.

Si tenemos en cuenta la información expuesta en la gráfica anterior podemos constatar que, 13 de los 29 participantes $(44,82 \%)$ escogieron la opción B. Con una diferencia considerable, 5 sujetos $(17,24 \%$ del total) seleccionaron la alternativa $E$ y otros 5 la opción F. Por otra parte, 4 participantes $(13,79 \%)$ han escogido la alternativa $\mathrm{D}$; mientras que únicamente 2 encuestados $(6,89 \%$ del total) se identificaban con la opción C. Finalmente, indicar que ninguno de los participantes ha seleccionado la alternativa $A$, por lo que podemos intuir que no se han sentido reflejados en esta opción.

A continuación, vamos a profundizar en la elaboración de prácticas mirando vídeos y reconociendo emociones en los niños. Los datos de esta cuestión quedan reflejados en la siguiente gráfica.

\section{Elaboración prácticas emociones}

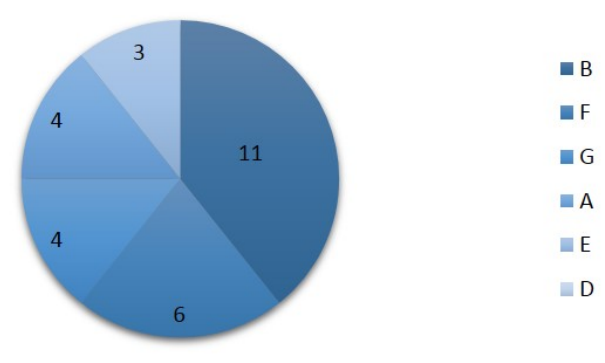

Figura 4. Datos sobre la elaboración de prácticas sobre las emociones.

A la pregunta relacionada con la elaboración de prácticas mirando vídeos y reconociendo emociones en los niños (práctica individual y grupal con anuncios de publicidad), los sujetos podían seleccionar entre diferentes opciones de respuesta entre las que se encuentran:

A.- Me ha ayudado a reconocer mejor las 5 emociones y sus combinaciones en los niños.

B.- Me ha ayudado a reconocer lo anterior y también qué tipo de inteligencia múltiple tiene cada niño (por ejemplo, si le emociona mucho la música esto sea indicio de que tiene inteligencia musical)

C.- Me ha ayudado a identificar mis emociones.

D.-Me ha ayudado a identificar mis emociones y reconocer que las tengo.

E.- Me ha ayudado a identificar, reconocer y gestionar mejor mis emociones.

F.- Me ha ayudado en todo lo anterior.

G.- Ninguna de las respuestas anteriores es mi caso. 
Si atendemos a los datos que aparecen en la gráfica $n^{\circ} 4$, la opción más escogida por los participantes en esta investigación ha sido la B seleccionada por 11 de 29 sujetos (37,93\% del total). La segunda alternativa más señalada por los encuestados ha sido la F elegida por 6 sujetos (20,68\%). Por otra parte, 4 de los 29 participantes $(13,80 \%)$ escogieron la alternativa A; otros $3(10,34 \%)$ señalaron la respuesta $E$. Sin embargo, 4 seleccionaron la opción G. Finalmente, sólo 1 participante $(3,44 \%)$ se sintió identificado con la respuesta $\mathrm{D}$. Del mismo modo, ningún sujeto ha seleccionado la opción $\mathrm{C}$ por lo que podemos constatar que no se han sentido reconocidos con esta alternativa. A la pregunta si de ti dependiera la obligatoriedad en las escuelas, ¿consideras que la inteligencia emocional es un factor clave en la educación de los niños? Por unanimidad la muestra ha contestado afirmativamente que sí.

A continuación, vamos a conocer la opinión de los estudiantes respecto a sí suelen ser una persona en la que confiar y a la que acuden los amigos en busca de consejo (Inteligencia Emocional), esta información queda recogida en la figura $n^{\circ} 5$ que mostramos seguidamente.

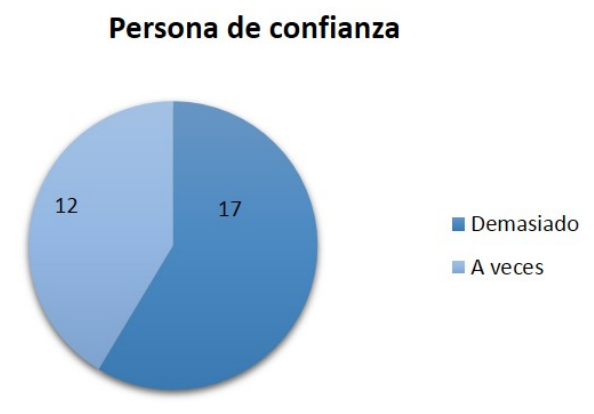

Figura 5. Datos sobre si se consideran personas de confianza.

A la pregunta relacionada con si se considera una persona en la que confiar y a la que acuden los amigos en busca de consejo (Inteligencia emocional), los sujetos tenían varias opciones de respuesta: sí, no y a veces. Más concretamente, 17 sujetos $(58,62 \%)$ seleccionaron la opción ¡Demasiado!, mientras que 12 (41,37\%) señalaron la opción "a veces". Como podemos comprobar existe una diferencia importante entre ambas alternativas.

Seguidamente, vamos conocer cuántos amigos tienen los sujetos participantes, haciendo especial énfasis en la inteligencia social. La información recogida queda ilustrada en la siguiente gráfica $n^{\circ} 6$.

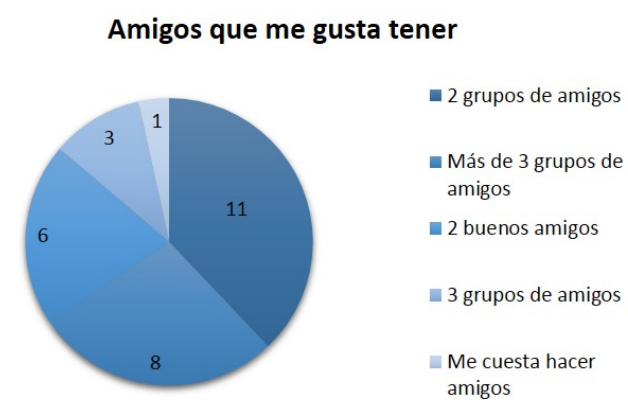

Figura 6. Datos sobre los amigos que les gusta tener. 
A la pregunta relacionada con el número de amigos que les gusta tener (Inteligencia social), los sujetos podían seleccionar entre diferentes opciones de respuesta entre las que se encuentran:

A.- Solamente dos mejores amigos.

B.- Dos grupos de amigos (los del Instituto y los de clase)

C.- Tres grupos de amigos (los del cole, los del Instituto y los de clase)

D.- Más de tres grupos de amigos (los de la guardería, los del cole, los del veraneo, los del Instituto, los de clase, los de alguna afición, los de la peña, etc.)

Más concretamente, y teniendo en cuenta la información que aparece en la gráfica $\mathrm{n}^{\circ} 9$, podemos afirmar que 11 de los 29 participantes $(37,93 \%)$ han señalado la opción B de dos grupos de amigos, 8 sujetos $(27,58 \%)$ han marcado la alternativa D esto es más de tres grupos de amigos. Por otra parte, 6 encuestados $(20,69 \%)$ se identificaron con la opción A solamente dos amigos; mientras que $3(10,34 \%)$ escogieron la alternativa $\mathrm{C}$, tres grupos de amigos. Finalmente, un único estudiante $(3,44 \%)$ se identificó con la opción "me cuesta hacer amigos" que no aparecía entre las alternativas a elegir en el cuestionario. No obstante, hemos considerado que era un dato relevante y significativo. De la muestra de 29 participantes 7 miembros son demasiado selectivos o tienen dificultades para hacer amigos.

Finalmente, se les preguntó sobre su preocupación por la inteligencia emocional. Más concretamente, se les cuestionó acerca de si habían realizado -o no- un test de inteligencia emocional. Las respuestas quedan reflejadas en la siguiente gráfica.

\section{Preocupación por la inteligencia emocional}
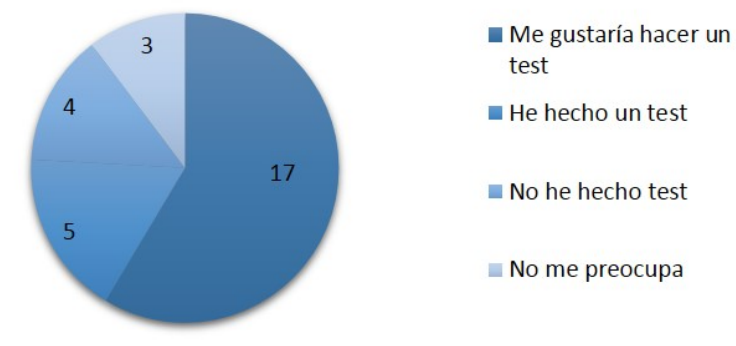

Figura 7. Datos sobre la preocupación por su Inteligencia Emocional.

A la pregunta relacionada con la realización o no de un test de inteligencia emocional para saber qué grado han tenido, los sujetos podían seleccionar entre 2 opciones de respuesta. Aunque como veremos en el proceso de análisis e interpretación de resultados, los estudiantes identificaron más alternativas. La opción "sí he hecho un test" fue señalada por 5 de los 29 encuestados (17,24\%), mientras que la alternativa "me gustaría hacer un test" fue marcada por 17 de los 29 $(58,62 \%)$. Como vemos, existe una diferencia importante entre los que realmente han hecho un test para conocer su inteligencia emocional y aquellos que tienen la intención de hacerlo o les gustaría hacerlo. Por el contrario, han sido 4 sujetos $(13,80 \%)$ los que se han identificado con la opción "no he hecho un test", mientras 
que 3 encuestados (10,34\%) indican "que no les preocupa". En este sentido, podemos confirmar que son menos los estudiantes que no han hecho un test o que no les preocupa hacerlo que aquellos que tienen interés por hacer el test o que realmente ya lo han realizado. Si tenemos en cuenta la información recogida en este segundo bloque de cuestiones podemos resaltar que un $38 \%$ de los alumnos aseguran que les ha ayudado a reconocer lo anterior y también qué tipo de inteligencia múltiple tiene cada niño (por ejemplo si le emociona mucho la música esto sea indicio de que tiene inteligencia musical); del mismo modo un casi un $59 \%$ de los estudiantes se consideran una persona de confianza y preocupados por su Inteligencia Emocional, incluso aseguran que les gustaría hacer un test, mientras que un $38 \%$ aproximadamente, confirma que tiene dos grupos de amigos.

Finalmente, en el cuestionario se les ofreció a los participantes una pregunta abierta para comentar o sugerir qué les hubiera gustado hacer más sobre inteligencia emocional, como es lógico al ser una respuesta libre con datos cualitativos hemos tenido que categorizar la información. Más concretamente, se han realizado 4 categorías: una primera en la que los estudiantes identifican que les hubiese gustado realizar pruebas relacionadas con Inteligencia Emocional; una segunda, en la que señalan que les hubiese gustado hacer más actividades vinculadas con la Inteligencia Emocional; una tercera, en la que resaltan la importancia de la Inteligencia Emocional y finalmente, una última categoría en la que indican la necesidad de saber más sobre este tipo de Inteligencia. Respecto a la primera categoría, si tomamos como referente las aportaciones de los sujetos podemos señalar que 7 de los 29 participantes (24\%) señalan que les hubiese gustado haber realizado más pruebas relacionadas con la Inteligencia Emocional. Concretamente, mencionaban que les hubiera gustado:

- (...) hacer pruebas de inteligencia emocional a los niños de un colegio.

- (...) haber trabajado más el tipo de inteligencia emocional.

- (...) haber podido hacer test para conocer nuestra inteligencia emocional y así poder gestionarla mejor en el futuro.

En cuanto a la segunda categoría podemos resaltar que, en ésta los estudiantes reconocen la importancia de realizar actividades relacionadas con este tipo de inteligencia. En este sentido, afirmaban que:

- La importancia de la inteligencia emocional en la comunicación y en las relaciones con los demás.

- Me encantaría que las emociones formarán parte de la distribución de materia en las escuelas y colegios infantiles porque desde ahí se pueden gestionar el desarrollo del aprendizaje para conseguir mejores resultados académicos y no académicos.

- Me hubiera gustado hacer una actividad que me ayudara más a conocer esta inteligencia, así como a definirla y trabajarla en mi misma, pues considero que es primordial para luego saber trabajarla en los niños.

La tercera categoría estaría relacionada con la importancia de la Inteligencia Emocional. Así pues, los estudiantes reconocían que:

- Me hubiera gustado hacer más actividades prácticas sobre la inteligencia emocional en el aula, porque la considera importante. 
- Tenemos que formar personas con corazón y no máquinas de trabajo productivo. Yo dedicaría me docencia a esta parte tan importante para descubrir cuáles son los talentos ocultos de cada niño y potenciarlo al máximo para que así pueda hacer uso de ello en un futuro y su vida pueda ser tranquila y feliz.

- Me hubiera gustado conocer más en profundidad algún programa que se esté llevando a cabo en escuelas de primaria sobre educación de la inteligencia emocional.

Por otra parte, algunos estudiantes señalaban algunas de las dificultades de poder llevar a cabo proyectos en el aula en los que se trabaje la IE. En este sentido, constataban que:

- La inteligencia emocional es bastante difícil de trabajar en estas edades y más con las horas lectivas de las que se disponen, por lo que creo que sólo sería necesario comentar la teoría y recomendar a los alumnos que ellos por su cuentan profundicen en el aspecto.

- Teniendo en cuenta que en modalidad semipresencial no se pueden llevar a cabo tantas actividades como en modalidad presencial,

Además, en cuanto a los resultados cualitativos recogidos de forma general tras la observación participante en clase, se descubrió que los alumnos reconocían a la inteligencia emocional como una esfera importante de beneficio personal y reconocían no haber tenido ninguna formación al respecto ni para identificar emociones, ni para expresarlas, ni para gestionarlas en anteriores niveles educativos. En su mayoría reconocían que debían mejorar al respecto sabiendo el resultado del test de inteligencia emocional (de autoevaluación). Asimismo, reconocían que debían mejorar en otra esfera, la social que está muy conectada con la inteligencia emocional: hacer más amigos y expresar más sus emociones como forma de autorregularse.

\section{DISCUSIÓN}

Tanto por los datos cualitativos como por los datos cualitativos de estos estudios de casos, se percibe un creciente interés por el conocimiento de la inteligencia emocional y su aplicación en los futuros maestros de educación infantil y primaria cuando se les presenta esta realidad y se les hace aplicable. Este hecho se puede poner en conexión con otros resultados de investigaciones anteriores de Peña puesto que en anteriores investigaciones se descubre una predisposición buena del alumnado universitario ante la inteligencia emocional, así como del profesorado (Suberviola, 2011). Asimismo, las conclusiones de estos estudios de casos se pueden poner en consonancia con la conveniencia de la inteligencia emocional para universitarios (Niño, García y Caldevilla, 2017; Morales, Giménez y Morales, 2018). Así como la necesidad de esta formación requerida tanto por competencia comunicacional como por superar los conflictos detectados en la propia convivencia en el aula (Sánchez Cobarro, 2016; Mira, Parra y Beltrán, 2017). Si cabe, se descubre la limitación de la interacción cara a cara para desarrollar mejor la expresión de la inteligencia emocional en los casos de la enseñanza semipresencial con respecto a la educación presencial donde es posible el encuentro cara a cara y el trabajo en sociedad del aula de forma corporal. En el caso de la enseñanza 
semipresencial se puede acudir al uso de vídeos para la identificación emocional y al estudio de casos para resolver casos de gestión emocional.

\section{CONCLUSIONES}

Así pues, entre las conclusiones menores más relevantes de estos estudios de casos las que podemos llegar tras concluir esta parte de la investigación es que la participación ha sido mayoritariamente femenina $(86,2 \%)$, con edades comprendidas entre los 23-50 años (72\%). Como podemos observar son personas con grado de madurez alto dada la edad en la que se encuentran cursando estudios universitarios, lo que probablemente le incite a conocer o querer saber más sobre su propia Inteligencia Emocional. En este sentido, son alumnas del Grado de Educación Infantil, de la modalidad semipresencial. Los resultados cuantitativos muestran que las alumnas tienen interés en la inteligencia emocional, tanto para su autoconocimiento como para su preparación profesional, cuestión que fue constatada asimismo mediante la observación participante en el aula y por los comentarios cualitativos. Por otra parte, cuando se les ha preguntado sobre el diario del clima de aula un 20 de la muestra considera que realizar esta tarea los ha llevado a observar más que antes a sus compañeros, las emociones de estos y conocer un poco más las suyas personales. Del mismo modo, un $45 \%$ de los estudiantes manifiesta que si el profesor identifica las emociones de los alumnos favorece que cada uno pueda percibir y entender sus emociones y como éstas son contagiadas al resto del grupo. Más concretamente, exponen el ejemplo de cómo se sienten ante un examen y cómo esta sensación/ emoción se transmite en el grupoclase. Asimismo, un $38 \%$ del alumnado considera que realizar prácticas en las que se perciben las emociones les ayuda a reconocerlas. Por otro lado, cuando se les ha preguntado por su situación personal el $58 \%$ de los participantes consideran que son una persona de confianza con la que puedes mostrar tus emociones. Más concretamente, cuando se les cuestiona sobre los amigos que les gusta tener existe una disparidad en las respuestas, ya que un 38\% considera que tiene 2 grupos de amigos (Instituto y la clase actual) y un $27,5 \%$ opina que tienen hasta 3 grupos de amigos (colegio, instituto y clase actual). Finalmente, a cuestiones relacionadas con su Inteligencia Emocional, un 58\% confirma que le gustaría hacer un test para conocer su IE, mientras que únicamente un $18 \%$ de la muestra ha realizado una prueba sobre IE aunque se les ofreció la oportunidad. En este sentido, resulta significativo, que la mayoría de los participantes no habían realizado un test que midiera su inteligencia emocional, y sin embargo sí que mostraban un gran interés por conocer estos aspectos en sí mismos para poder posteriormente aplicarlos en el aula. Quizás se deba a falta de atención a las instrucciones del profesor que lo facilitó o la falta de recursos de búsqueda para encontrar test gratuitos online si tenían tanto interés.

Así pues, los participantes destacan que en el aula en este proyecto se trabajan aspectos como la identificación emocional, lo que les ayuda a identificar sus propias emociones. En cuanto a los datos cualitativos recogidos a través de la pregunta abierta es de destacar su ansia por aprender a saber identificar emociones, autoconocerse mejor a través de test, la importancia que le otorgan sin dudarlo y por último el interés por profundizar más en esta cuestión. En cuanto a la información cualitativa recogida por la profesora destaca el interés que mostraron sobre la 
cuestión para conocer la inteligencia emocional de forma más práctica y aplicada para saber autogestionarse mejor y conocerse a sí mismos, así como la necesidad de desarrollar más la empatía con respecto a las emociones de los demás, y, conectada a esta idea además saber hacer amigos, de modo que aprendan a disponer de más grupos de amigos entendiendo los beneficios de la sociabilidad en su desarrollo personal y la necesidad de ser expertos en humanidad si se dedican a la docencia.

En definitiva, este estudio denota que, a pesar de haber aplicado un proyecto innovador de inteligencia emocional, esta competencia emocional fue apenas introducida por las actividades que se realizaron y quedaba mucho por hacer por las carencias en cuanto a la identificación, expresión y gestión que reconoció el alumnado de estos casos. Asimismo, fue reconocido por los alumnos que debían aumentar su capacidad social. De este modo, los alumnos de Educación se mostraron perceptivos y pidieron más actividades y formación práctica sobre la inteligencia emocional. Se estimó conveniente realizar un proyecto de innovación posterior. Esto nos hace pensar que, aunque existe en el ámbito educativo tanto por parte de profesorado como de alumnado una apuesta por el desarrollo de estas competencias socioemocionales todavía queda mucho camino por recorrer y que son convenientes formación para docentes y asimismo desarrollar intervenciones docentes concretas y prácticas en este sentido desde los niveles iniciales para los discentes. Además, hay que tener en cuenta que el mejor aprendizaje es el vicario y que el profesorado que forma a los alumnos de Educación debe vivirlo y no saberlo explicar teóricamente. De este modo estimamos que es más fácil realizar el aprendizaje por imitación o modelado. Estimamos, además, que la realización de investigaciones similares a la que nos ocupa, no solo permite acercar la inteligencia emocional a los docentes, sino que en un futuro también podrá establecer las bases de un sistema educativo que facilite el desarrollo personal y profesional de todo el colectivo implicado en la enseñanza y favorecer positivamente el bienestar emocional individual para repercutir además en la cultura y en el estilo de vida de la sociedad.

\section{REFERENCIAS}

\section{Publicaciones periódicas:}

Arís Redó, N. (2010). La educación emocional y la comunicación escolar. Vivat Academia, (113), 79-87.

Bar-On, R. (2006). The Bar-On model of Emotional-Social Intelligence. Psicothema, (18)1, 13-25.

Bisquerra, R. (2000). Educación emocional y bienestar. Barcelona: Praxis.

Bisquerra, R. (2003). Educación emocional y competencias básicas para la vida. Revista de Investigación Educativa (RIE), 21(1), 7-43. 
Extremera N. y P. Fernández-Berrocal (2004). El uso de las medidas de habilidad en el ámbito de la Inteligencia emocional. Boletín de Psicología, (80), 59-67.

Extremera, N. y Fernández-Berrocal, P. (2003). La inteligencia emocional en el contexto educativo: hallazgos científicos de sus efectos en el aula. Revista de Educación, (332), 97-116.

Extremera, N. y Fernández-Berrocal, P. (2003). La inteligencia emocional: Métodos de evaluación en el aula. Revista Iberoamericana de Educación, (30), 1-12.

Fernández de Bobadilla, G. W., Peña Acuña, B. y Parra Meroño, M. C. (2014). La inteligencia social: Investigación en maestros de infantil y primaria. En E. Bernad Monferrer, (coord.), Actualización de los nuevos sistemas educativos (pp. 533549). Madrid: ACCI.

Fernández-Berrocal, P. y Extremera, N. (2002). La inteligencia emocional como una habilidad esencial en la escuela. Revista Iberoamericana de Educación, (29), 1-6.

Fernández-Berrocal, P. y Extremera, N. (2005). La inteligencia emocional y la educación de las emociones desde el Modelo de Mayer y Salovey. Revista Interuniversitaria de Formación de Profesorado, 19(3), 63-93.

Gardner, H. (2001). La inteligencia reformulada. Las inteligencias múltiples en el siglo XXI. Barcelona: Paidós.

Goleman, D. (2004). Inteligencia Emocional. Barcelona: Kairós.

López de la Serna, A. Castaño-Garrido, C. (2018). Estudio de las emociones, el aprendizaje autorregulado y la motivación en un curso SPOC de la Universidad del País Vasco (UPVI EHU), International Journal of Educational Research and Innovation (IJERI), (10), 299-316.

Mayer, J. D. y Salovey, P. (1997). What is emotional intelligence? En P. Salovey y D. Sluyter (Eds.), Emotional development and emotional intelligence: implications for educators, (pp. 3-31). New York: Basic Books.

Morales Rodríguez, F. M., Giménez Lozano, J. M., y Morales Rodríguez, A. M. (2018). Relaciones entre autoeficacia emprendedora y otras variables psicoeducativas en universitarios, European Journal of investigation in health, psicology and education, 8(2). doi: https://doi.org/10.30552/ejihpe.v8i2.247 Recuperado de https://formacionasunivep.com/ejihpe/index.php/journal/article/view/247

Mira Agulló, J. G.; Parra Meroño, M. C. y Beltrán Bueno, M. A. (2017). Educación emocional en la universidad: propuesta de actividades para el desarrollo de habilidades sociales y personales. Revista Vivat Academia, (139), 1-17. doi: http://doi.org/10.15178/va.2017.1-17

Recuperado de http://www.vivatacademia.net/index.php/vivat/article/view/1019 
Niño González, J. I., García García, E. y Caldevilla Domínguez, D. (2017). Inteligencia emocional y educación universitaria: una aproximación Revista de Comunicación de la SEECI, (43), 15-27. doi: http://dx.doi.org/10.15198/seeci.2017.43.15-27 Recuperado de http://www.seeci.net/revista/index.php/seeci/article/view/486

Pedrera, M. I. y Revuelta, F. I. (2016). Estudio de la escala "reparación emocional" en los alumnos de prácticas externas del Grado de Educación Social, International Journal of Educational Research and Innovation (IJERI), (6), 71-82.

Peña Acuña, B., Díez Frutos, M. y García Gómez, M. (2012). La convivencia de la Inteligencia Emocional por parte de los maestros de Infantil y Primaria. En B. Peña Acuña (coord.), Desarrollo Humano (pp. 15-48). Madrid: Visión Libros.

Peña Acuña, B. y Muñoz Sánchez, P. (2014). Evaluación mediante la observación participante de una metodología de trabajo en equipo para el desarrollo de competencias básicas de la inteligencia social. En J. J., González Ortiz, F. J. Sánchez Marín y M. A. Castejón, Innovación, evaluación y universidad (pp. 4660). Murcia: Fundación Universitaria San Antonio.

Peña Acuña, B. (2018). Comunicación interpersonal. Muñoz Sánchez, P. y Vitón de Antonio, $\mathrm{M}^{\circ}$. J. (Coord.) Comunidad, desarrollo y escenarios educativos emancipatorios. (pp.139-148). Murcia: Compobell.

Salovey, P. \& Mayer, J. D. (1990). Emotional Intelligence. Imagination, Cognition and Personality, 9(3), 185-211.

Sánchez, F. J., et al. (2015). Desarrollo de la Inteligencia Social a través del Aprendizaje Cooperativo en alumnos de Grados universitarios y Ciclos Formativos de Grado Superior. En J. J. González y A. González (Eds.). La Universidad como comunidad de innovación y cambio (pp. 174-182). Murcia: Fundación Universitaria San Antonio.

Sánchez Cobarro, P. del H. (2016). Emociones y comunicación. En Sánchez, García, Gómez, et al. (Coord.). Bienestar Emocional (pp. 39-58). Madrid: Dykinson.

Suberviola Ovejas, I., (2011). Autopercepción del profesorado sobre su educación emocional. Revista Vivat Academia, 14(117E), 1154-1167.

\section{AUTORAS:}

\section{Beatriz Peña Acuña}

Profesora con acreditación de Profesor Titular en la Universidad de Huelva. Formación interdisciplinar en España, EE.UU. e Inglaterra. Premio extraordinario de doctorado por la Universidad de Alicante (2012). Tiene un sexenio de investigación reconocido. Imparte docencia en la Facultad de Educación. Su producción científica en varios idiomas es prolífica y le apasiona divulgar la ciencia. Ha recibido diversos 
reconocimientos, entre estos, Honoris Causa por UFA (Rusia), President's Life Time Achievement Award (EE.UU.), Real Sociedad Menéndez Pelayo, etc.

beatriz.pa@dfilo.uhu.es

https://uhu.academia.edu/BeatrizPe\%C3\%B1aAcu\%C3\%B1a

\section{María Luisa García Hernández}

Licenciada en Pedagogía. Máster en tecnología educativa: aprendizaje virtual y gestión del conocimiento. Doctora en Educación. Experta en Didáctica y en Enseñanza Superior. Actualmente es profesora en el Departamento de Didáctica y Organización Escolar perteneciente a la Facultad de Educación en la Universidad de Murcia. Su producción científica sobre estas cuestiones es prolífica.

luisagarcia@um.es

https://www.researchgate.net/profile/Maria Garcia Hernandez4 